\title{
Steep extinction towards GRB 140506A reconciled from host galaxy observations: Evidence that steep reddening laws are local $\star, \star \star$
}

\author{
K. E. Heintz ${ }^{1,2}$, J. P. U. Fynbo ${ }^{2}$, P. Jakobsson ${ }^{1}$, T. Krühler ${ }^{3}$, L. Christensen ${ }^{2}$, D. Watson ${ }^{2}$, C. Ledoux ${ }^{4}$, P. Noterdaeme ${ }^{5}$, \\ D. A. Perley ${ }^{6,2}$, H. Rhodin ${ }^{2}$, J. Selsing ${ }^{2}$, S. Schulze ${ }^{7}$, N. R. Tanvir ${ }^{8}$, P. Møller ${ }^{9}$, P. Goldoni ${ }^{10}$, \\ D. $\mathrm{Xu}^{11}$, and B. Milvang-Jensen ${ }^{2}$
}

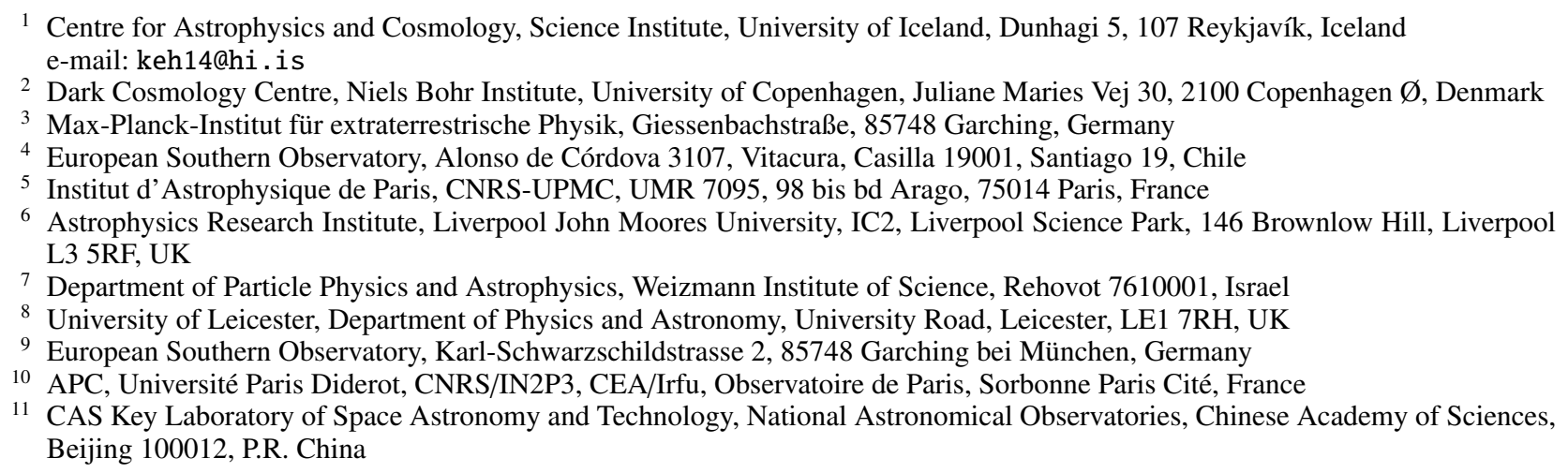

Received 27 February 2017 / Accepted 20 March 2017

\begin{abstract}
We present the spectroscopic and photometric late-time follow-up of the host galaxy of the long-duration Swift $\gamma$-ray burst GRB 140506A at $z=0.889$. The optical and near-infrared afterglow of this GRB had a peculiar spectral energy distribution (SED) with a strong flux-drop at $8000 \AA$ (4000 Å rest-frame) suggesting an unusually steep extinction curve. By analysing the contribution and physical properties of the host galaxy, we here aim at providing additional information on the properties and origin of this steep, non-standard extinction. We find that the strong flux-drop in the GRB afterglow spectrum at $<8000 \AA$ and rise at $<4000 \AA$ (observers frame) is well explained by the combination of a steep extinction curve along the GRB line of sight and contamination by the host galaxy light at short wavelengths so that the scenario with an extreme $2175 \AA$ extinction bump can be excluded. We localise the GRB to be at a projected distance of approximately $4 \mathrm{kpc}$ from the centre of the host galaxy. Based on emission-line diagnostics of the four detected nebular lines, $\mathrm{H} \alpha, \mathrm{H} \beta,[\mathrm{O} \mathrm{II}]$ and [O III], we find the host to be a modestly star forming $\left(S F R=1.34 \pm 0.04 M_{\odot} \mathrm{yr}^{-1}\right)$ and relatively metal poor $\left(Z=0.35_{-0.11}^{+0.15} Z_{\odot}\right)$ galaxy with a large dust content, characterised by a measured visual attenuation of $A_{\mathrm{V}}=1.74 \pm 0.41 \mathrm{mag}$. We compare the host to other GRB hosts at similar redshifts and find that it is unexceptional in all its physical properties. We model the extinction curve of the host-corrected afterglow and show that the standard dust properties causing the reddening seen in the Local Group are inadequate in describing the steep drop. We thus conclude that the steep extinction curve seen in the afterglow towards the GRB is of exotic origin and is sightline-dependent only, further confirming that this type of reddening is present only at very local scales and that it is solely a consequence of the circumburst environment.
\end{abstract}

Key words. gamma-ray burst: general - gamma-ray burst: individual: GRB 140506A - dust, extinction - galaxies: individual: GRB 140506A host

\section{Introduction}

Gamma-ray bursts (GRBs) have proven to be valuable tools to probe the interstellar medium (ISM), chemical enrichment and dust content of star-forming galaxies both in the local Universe

\footnotetext{
$\star$ Based on observations carried out under programme IDs 095.D0043(A, C) and 095.A-0045(A) with the X-shooter spectrograph and the FOcal Reducer and low dispersion Spectrograph 2 (FORS2) installed at the Cassegrain Very Large Telescope (VLT), Unit 2 - Kueyen and Unit 1 - Antu, respectively, operated by the European Southern Observatory (ESO) on Cerro Paranal, Chile.

$\star \star$ The reduced spectra (FITS files) are only available at the CDS via anonymous ftp to cdsarc.u-strasbg. fr (130.79.128.5) or via http://cdsarc.u-strasbg.fr/viz-bin/qcat?J/A+A/601/A83
}

and out to high redshifts (e.g. Jakobsson et al. 2004; Fynbo et al. 2006, 2009; Prochaska et al. 2007; Li 2008; Ledoux et al. 2009; Gehrels et al. 2009; Schady et al. 2012; De Cia et al. 2013; Thöne et al. 2013; Sparre et al. 2014; Hartoog et al. 2015). Specifically, the optical and near-infrared extinction curves seen toward GRB afterglows reveal that they typically can be welldescribed by similar prescriptions to that of the Milky Way (MW) and the Small and Large Magellanic Clouds (SMC and LMC) (see e.g. Schady et al. 2010; Covino et al. 2013; Japelj et al. 2015). In rare cases, however, the afterglows appear to show flat (also known as grey) extinction curves (Savaglio \& Fall 2004; Perley et al. 2008; Friis et al. 2015) or at the opposite extreme, very steep extinction curves. In the latter case, a prime example is seen towards the afterglow of 
GRB 140506A (Fynbo et al. 2014) but also reported in the spectral energy distribution (SED) of the afterglow of GRB 070318 (Fynbo et al. 2009; Watson 2009) and bluewards of the observed $2175 \AA$ bump in GRB 080605A (Zafar et al. 2012; Krühler et al. 2012).

This paper reports on the spectroscopic and photometric latetime follow-up of the host galaxy of GRB 140506A at $z=$ 0.889 . This GRB was first detected by Swift on 2014 May 6, 21:07:36 UT (Gompertz et al. 2014). At 8.8 and $33 \mathrm{~h}$ postburst, Fynbo et al. (2014) acquired spectra with the X-shooter spectrograph (Vernet et al. 2011) which, together with extensive imaging from the Gamma-Ray burst Optical and Near-infrared Detector (GROND; Greiner et al. 2008), revealed a peculiar optical and near-infrared afterglow and the first detections of He I* and Balmer line absorption in GRBs. In contrast to other GRB afterglow SEDs, which typically can be modelled with a simple power-law with weak SMC-like extinction, a strong, gradual flux drop is seen below $8000 \AA$ (4000 $\AA$ rest-frame, see Fig. 1) that can not be modelled by any of the extinction curves known from the Local Group. By analysing the host we here aim to better constrain the physical origin of this steep extinction curve.

The paper is structured as follows. In Sect. 2 we describe our observations and in Sects. 3 and 4 we describe how we constrained the properties of the host galaxy with special emphasis on the implications for the interpretation of the extinction derived from the afterglow spectroscopy. In Sect. 5 we summarise and conclude. Throughout the paper we assume a standard flat $\Lambda \mathrm{CDM}$ cosmology with $H_{0}=67.3 \mathrm{~km} \mathrm{~s}^{-1} \mathrm{Mpc}^{-1}, \Omega_{\mathrm{m}}=0.315$ and $\Omega_{\Lambda}=0.685$ (Planck Collaboration et al. 2014). Unless otherwise stated, all magnitudes are given in the AB (Oke 1974) magnitude system.

\section{Observations and data reduction}

Based on the peculiar (and temporally varying) SED of the optical and near-infrared afterglow of GRB 140506A, acquired at two epochs at $\Delta t=8.8 \mathrm{~h}$ and $\Delta t=33 \mathrm{~h}$ after the burst, respectively, we decided to observe the host galaxy of the GRB to examine the nature of this in more detail than what can be extracted from the afterglow spectra.

\subsection{FORS2 imaging}

We acquired late-time photometry with the FOcal Reducer and low dispersion Spectrograph 2 (FORS2; Appenzeller et al. 1998) instrument at the ESO/VLT on 2015 April 29 and June 16. We obtained images of the field of GRB 140506A using the $u_{\mathrm{HIGH}}$, $g_{\text {HIGH }}, R_{\text {SPECIAL }}$ and $I_{\text {BESS }}$ broadband filters (henceforth, these will be referred to as simply the $u$-, $g$-, $R$ - and $I$-band, respectively). In Table 1 a log of the FORS 2 observations is provided together with the measurements of the magnitude of the host galaxy in each filter.

The photometric calibration of the observations in the $u$ - and $g$-bands was done using zeropoints measured from reference stars observed at similar airmasses on the nights of the observations of the host galaxy. For the $R$ - and $I$-bands we use the zeropoints reported in the ESO archive for the night of observation. All images were taken following a dithering pattern and were bias-subtracted and flat-fielded using standard IRAF (Tody 1993) routines. The images acquired in April were taken under excellent conditions with a median seeing of 0 ". 61 , where for the images obtained in June we report a median seeing of $00^{\prime \prime} 69$. To measure the magnitude of the host we performed aperture

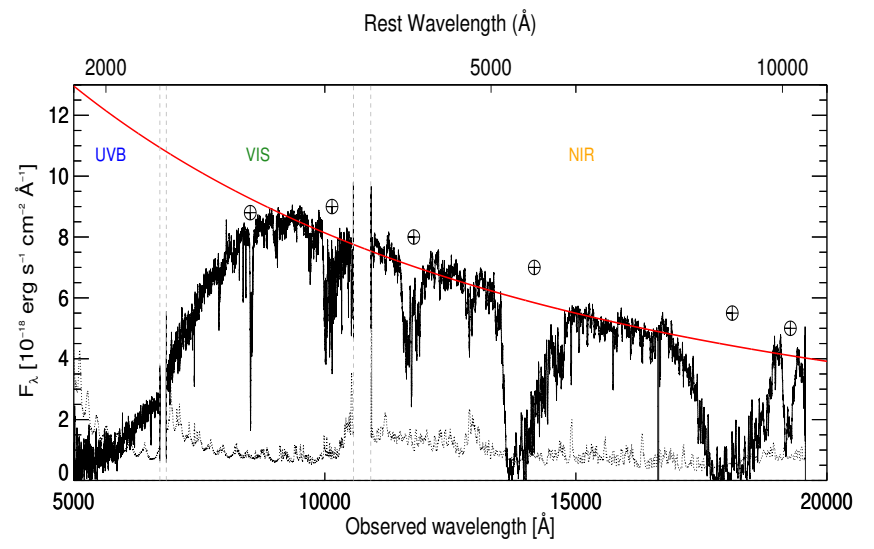

Fig. 1. X-shooter spectrum of the first epoch afterglow (identical to the top panel in Fig. 4 of Fynbo et al. 2014). Regions affected by telluric absorption are marked by the corresponding symbol. The error spectrum is plotted as the dotted line. Overplotted is the best-fit power law following $F_{\lambda} \propto \lambda^{-\beta}$, fitted to the afterglow continuum at $>8000 \AA$ shown by the red solid line.

photometry of the source in the combined frames of each filter using standard MIDAS routines.

\subsection{Spitzer/IRAC observations}

The position of GRB 140506A was observed by Spitzer/IRAC in the $3.6 \mu \mathrm{m}$ filter as part of the extended Swift/Spitzer GRB Host Galaxy Survey (SHOALS; Perley et al. 2016, GO program 11116) on 2015 July 4, with a total integration time of $2400 \mathrm{~s}$. We downloaded the reduced imaging (PBCD) from the Spitzer Heritage Archive. The area close to the GRB host galaxy is affected by a weak pulldown artifact from a nearby star; we fixed this manually by adding a constant term to the affected columns. The host galaxy is faint but clearly detected in this band, and there is no strong blending from any nearby sources. We measure photometry of the galaxy again using standard aperture photometry techniques using the zeropoints in the IRAC handbook for the absolute calibration.

\subsection{X-shooter spectroscopy}

To supplement the FORS2 and Spitzer imaging we acquired additional spectra centred on the host galaxy using the X-shooter spectrograph mounted at the ESO/VLT, covering a wavelength range of 3000-24 $800 \AA$ (3000-5600, 5500-10 200 and 10200 $24800 \AA$ for the UVB, VIS and NIR arm, respectively). The follow-up observations of this host were spread out over three nights, on 2015 May 15, June 15 and September 30. The reason for this was that we realised that the heliocentric velocity shift had placed the $\mathrm{H} \alpha$ line on top of a bright sky emission line in May and June. In September the velocity caused by the Earth's orbit was optimal for separating the $\mathrm{H} \alpha$ emission line away from the skyline.

The observations were carried out more than a year after the GRB and we can hence safely assume that there is no significant contribution from the afterglow to the measured fluxes. Extrapolating the temporal flux decay from the GROND afterglow observations suggests that the afterglow contributes less than $5 \%$ of the host galaxy flux at this point. The observations were all carried out under good conditions with a seeing between $\sim 0$ '.7-1'.2 at airmasses between 1.1 and 1.5. The observations in May were 
Table 1. Photometry of the host galaxy from FORS2 and Spitzer/IRAC imaging.

\begin{tabular}{ccccccc}
\hline \hline Date $(\Delta t)^{a}$ & Filter & Eff. wavelength & Exp. time & Avg. airmass & Seeing $^{b}$ & Mag $_{\mathrm{AB}}^{c}$ \\
\hline $2015-04-29(357.48 \mathrm{~d})$ & $u_{\mathrm{HIGH}}$ & $3610 \AA$ & $3 \times 500 \mathrm{~s}$ & 1.166 & $0^{\prime \prime} 62$ & $25.60 \pm 0.30$ \\
$2015-04-29(357.50 \mathrm{~d})$ & $g_{\mathrm{HIGH}}$ & $4700 \AA$ & $3 \times 300 \mathrm{~s}$ & 1.171 & $0^{\prime \prime} \cdot 60$ & $25.47 \pm 0.21$ \\
$2015-06-16(405.24 \mathrm{~d})$ & $R_{\text {SPECIAL }}$ & $6550 \AA$ & $5 \times 200 \mathrm{~s}$ & 1.323 & $0^{\prime \prime} 70$ & $25.16 \pm 0.19$ \\
$2015-06-16(405.26 \mathrm{~d})$ & $I_{\text {BESS }}$ & $7680 \AA$ & $5 \times 200 \mathrm{~s}$ & 1.280 & $0^{\prime \prime} 67$ & $24.46 \pm 0.20$ \\
$2015-07-04(423.12 \mathrm{~d})$ & IRAC 1 & $3.6 \mu \mathrm{m}$ & $2400 \mathrm{~s}$ & $\cdots$ & $\cdots$ & $23.88 \pm 0.30$ \\
\hline
\end{tabular}

Notes. ${ }^{(a)}$ The date of observations are listed with the number of days from the trigger in brackets. The time is calculated from the start of observations to the time of trigger. ${ }^{(b)}$ Measured from the full-width-at-half-maximum of non-saturated stellar sources on the stacked images. ${ }^{(c)}$ Magnitudes reported here have been corrected for the Galactic foreground reddening and will be used throughout our analysis.

Table 2. Spectroscopic observations of the host galaxy with X-shooter.

\begin{tabular}{cccc}
\hline \hline Date $(\Delta t)^{a}$ & Exp. time & Avg. airmass & Seeing $^{b}$ \\
\hline $2015-05-15(373.47 \mathrm{~d})$ & $4 \times 600 \mathrm{~s}$ & 1.198 & $0^{\prime \prime} \cdot 72$ \\
$2015-06-15(404.31 \mathrm{~d})$ & $4 \times 600 \mathrm{~s}$ & 1.184 & $0^{\prime \prime} 90$ \\
$2015-06-15(404.36 \mathrm{~d})$ & $4 \times 600 \mathrm{~s}$ & 1.173 & $0^{\prime \prime} 98$ \\
$2015-06-15(404.41 \mathrm{~d})$ & $5 \times 600 \mathrm{~s}$ & 1.268 & $0^{\prime \prime} 84$ \\
$2015-09-30(511.11 \mathrm{~d})$ & $4 \times 600 \mathrm{~s}$ & 1.233 & $1^{\prime \prime} 24$ \\
$2015-09-30(511.15 \mathrm{~d})$ & $4 \times 600 \mathrm{~s}$ & 1.332 & $1^{\prime \prime} 18$ \\
$2015-09-30(511.19 \mathrm{~d})$ & $4 \times 600 \mathrm{~s}$ & 1.492 & $0^{\prime \prime} .81$ \\
\hline
\end{tabular}

Notes. ${ }^{(a)}$ Same notation as in Table 1. ${ }^{(b)}$ The seeing is measured from the telescope guide probe.

carried out in a $4 \times 600$ s observing block $(\mathrm{OB})$, while in June and September we acquired three executions of $4 \times 600 \mathrm{~s}$ (except for the last $\mathrm{OB}$ in June where we acquired $5 \times 600 \mathrm{~s}$ ), following an ABBA nodding pattern. The slit was aligned with the parallactic angle, and for the multiple executions in June and September we re-aligned the slit between the three executions of the OBs. The slit widths were $1^{\prime \prime} 0,0, .9$ and $0 \prime .9$ in the UVB, VIS and NIR arm, respectively, for all observations. The details for the $\mathrm{X}$-shooter observations are all listed in Table 2. For this given setup the nominal instrumental resolution in the respective arms are $\mathcal{R}_{\mathrm{UVB}}=5100, \mathcal{R}_{\mathrm{VIS}}=8800$ and $\mathcal{R}_{\mathrm{NIR}}=5100^{1}(\approx 59,34$ and $59 \mathrm{~km} \mathrm{~s}^{-1}$, respectively). From a set of atmospheric emission lines in the VIS and NIR arms we measure resolutions of $\mathcal{R}_{\mathrm{VIS}}=9096\left(\approx 33 \mathrm{~km} \mathrm{~s}^{-1}\right)$ and $\mathcal{R}_{\mathrm{NIR}}=6977\left(\approx 43 \mathrm{~km} \mathrm{~s}^{-1}\right)$ at full-width-at-half-maximum (FWHM). These values were determined from sky-lines close to the detected emission lines, and will be used to correct for the intrinsic line widths.

For the X-shooter data reduction we followed the same procedure described in detail in Krühler et al. (2015), so in the following we will only briefly outline our strategy. First we used the ESO X-shooter pipeline (Goldoni et al. 2006; Modigliani et al. 2010) to produce a flat-fielded, rectified and wavelength-calibrated 2D spectrum for every frame in the UVB and VIS arm. For the NIR arm we produced two frames, one for every nod cycle. The resulting 2D frames are then sky-subtracted and rejected for cosmic rays using our own software. The frames are combined by shifting the individual frames and using a weighted average from the signal-to-noise ratio $(\mathrm{S} / \mathrm{N})$ to produce one single 2D frame for each arm. We then use a custommade Python code (again see Krühler et al. 2015) to perform optimal extraction on the final $2 \mathrm{D}$ frame to obtain the extracted

\footnotetext{
1 https://www.eso.org/sci/facilities/paranal/ instruments/xshooter/inst.html.
}

1D spectrum, using a running Moffat-profile fit to improve the $\mathrm{S} / \mathrm{N}$. All wavelengths reported are in vacuum and are corrected for the heliocentric velocity and the Galactic foreground reddening of $E(B-V)=0.082$ mag (Schlafly \& Finkbeiner 2011).

\section{Results}

\subsection{Locating the GRB explosion site}

An important piece of information concerning the nature of the steep extinction seen in the optical/near-infrared afterglow of GRB 140506A is the location of the explosion site within its host galaxy. In Fig. 2 we show the projected position of the explosion site relative to the host galaxy in the stacked $I$-band image obtained with VLT/FORS2. The exact location of the afterglow is determined by coaligning the host image with an image containing the afterglow using a set of reference stars common to both images. The respective centres of the afterglow and the host galaxy was determined by fitting a Gaussian profile to the image profiles. The semi-major axis of the host galaxy, derived from the FWHM of the fitted Gaussian profile, is $0.96(7.68 \mathrm{kpc}$ at $z=0.889)$. The derived position and its $3 \sigma$ astrometric error is shown by the white cross and white circle, respectively.

We derive an offset of $0.49 \pm 0.03(3.92 \pm 0.24 \mathrm{kpc}$ at $z=0.889$ ) from the projected position of the afterglow to the centre of the host and show that it originated in a faint region in the outskirts of the galaxy (while GRBs are on average found to explode in the brightest optical regions of their host galaxies, see e.g. Bloom et al. 2002; Fruchter et al. 2006; Lyman et al. 2017). We note that higher resolution is required to securely exclude high-surface brightness at the afterglow position that could be washed out due to ground-based seeing.

The column density of neutral hydrogen $\left(N_{\mathrm{HI}}\right)$ at the GRB explosion sites are typically measured from the broad, damped Lyman- $\alpha(\operatorname{Ly} \alpha)$ trough for GRBs at $z \gtrsim 1.8$. Due to the low redshift of this system, however, we are not able detect this absorption feature. From the Swift X-Ray Telescope (XRT) spectra, obtained in photon counting (PC) mode, an intrinsic column density of $N_{\mathrm{H}, \mathrm{X}}=6.7_{-1.3}^{+1.4} \times 10^{21} \mathrm{~cm}^{-2}$ is determined for this burst (see the Swift-XRT GRB spectrum repository ${ }^{2}$ ). This value is among the highest $10 \%$ of optically detected bursts (Fynbo et al. 2009), but is typical in Swift XRT-observed GRB afterglows (e.g. Campana et al. 2010; Starling et al. 2013; Buchner et al. 2017).

\footnotetext{
2 http://wWw.swift.ac.uk/xrt_spectra/
} 

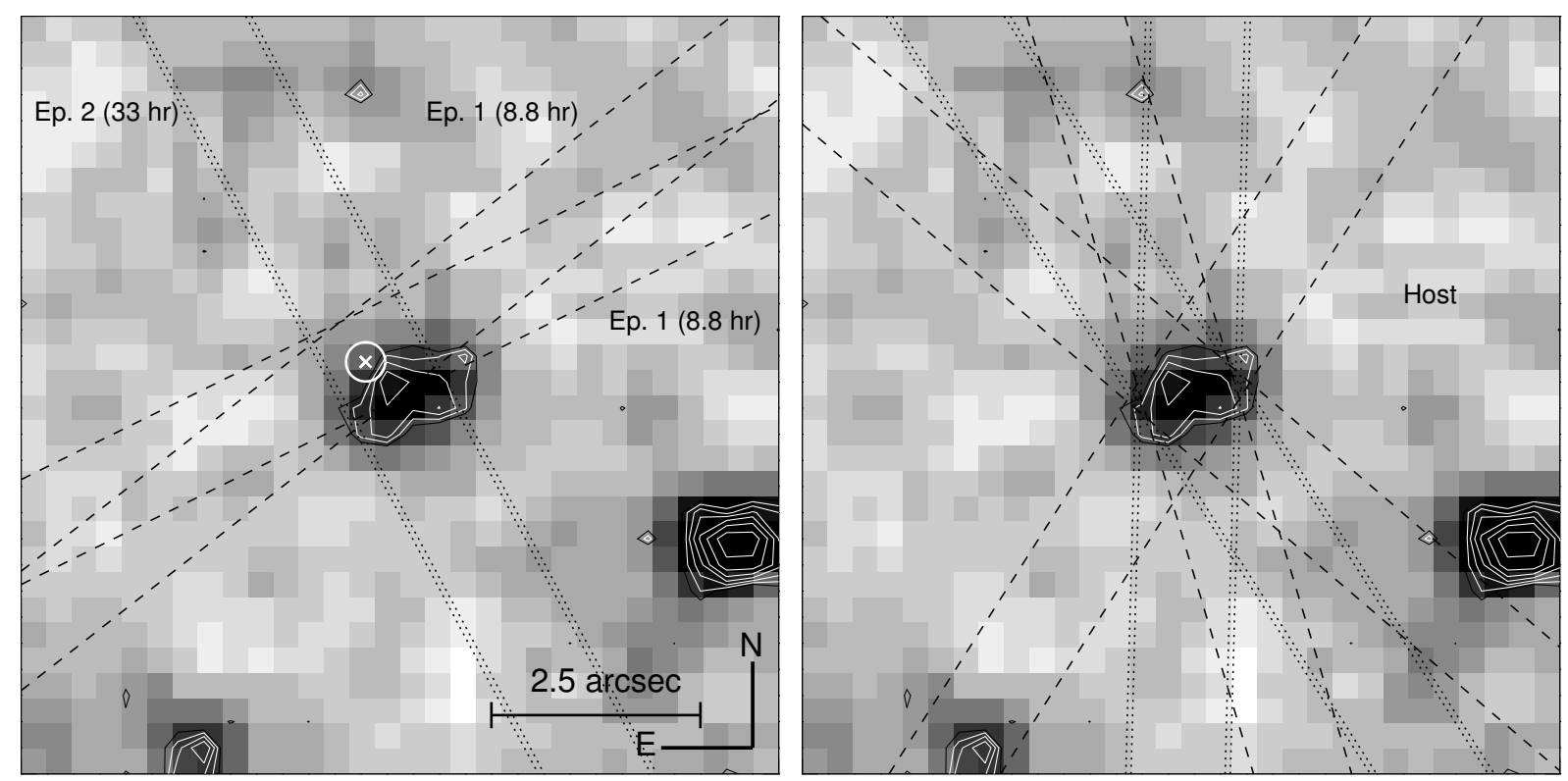

Fig. 2. Close-up of the field of GRB $140506 \mathrm{~A}$ and its host galaxy from the combined FORS2 I-band image $\left(7.75^{\prime \prime} \times 7.75^{\prime \prime}\right.$ in both panels). The position of the afterglow is offset $0 \prime \prime 49 \pm 0.03(3.92 \pm 0.24 \mathrm{kpc}$ at $z=0.889)$ from the host galaxy shown by the white cross with the $3 \sigma$ uncertainty on the position shown by the surrounding white circle. Schematics of the slit positions used to observe the afterglow and the host are shown during the first and second epoch (left panel) and from the late-time follow-up (right panel). The image has been smoothed to enhance the contrast and the white contours show the relative image flux levels.

\subsection{Excluding the $2175 \AA$ A extinction bump scenario}

The extreme flux-deficit below $<8000 \AA$ (observer frame) seen in the spectrum of the afterglow, is suggested by Fynbo et al. (2014) to be caused by either an extreme $2175 \AA$ bump feature in the extinction curve or due to multiple scattering of light in the nearby environment. The multiple scattering scenario, however, does not seem to be well substantiated in general and was indeed ruled out as the origin of the steep extinction seen towards the supernova SN 2014J (Johansson et al. 2017). The $2175 \AA$ extinction bump feature was motivated by the fact that the binned afterglow spectrum appeared to increase in flux below $\sim 4000 \AA$ which would be predicted if the absorption feature was indeed due to a bump in the extinction curve. Even though the $2175 \AA$ extinction bump is seen in some GRB afterglows, they are still rare with only four, robustly detected systems, GRBs 070802 (Krühler et al. 2008; Elíasdóttir et al. 2009), 080607 (Prochaska et al. 2009; Perley et al. 2011; Zafar et al. 2011), 080605 and 080805 (Zafar et al. 2012). However, none of these are as extreme as what is seen towards GRB 140506A.

To test the hypotheses of the $2175 \AA$ extinction bump, we compare the contribution of the host galaxy to the afterglow (see Fig. 3). Following the same procedure as was done for the afterglow we bin the spectrum of the host galaxy below $5000 \AA$ in $100 \AA$ bins to match that of the afterglow spectrum. It is evident that the rise seen in the afterglow spectrum is purely due to the afterglow being host-dominated below $4000 \AA$ in the first and second epoch. This then indicates that the scenario with the flux-drop being caused by an extreme $2175 \AA$ extinction bump feature can be safely excluded. This is further supported by the fact that such an extreme bump would be approximately three times as high as seen toward any line-of-sight in the Milky Way (Fitzpatrick \& Massa 2007; Fynbo et al. 2014). See Sect. 4.2 for a parametrization of the extinction curve of the host-corrected first epoch afterglow with the bump or "Drude"-profile removed.
Fitting the Fitzpatrick \& Massa (2007) extinction curve with the central wavelength of the bump set as a free parameter favours a centre at $2015 \AA$. It is, however, a poor fit and the associated bump width would be a factor of five larger than that typically probed in the Milky Way. This case then leads the way for a non-standard reddening which will have a crucial impact for our understanding of the properties of dust grains in the nonlocal Universe. We will discuss this more in Sect. 4.

\subsection{Underlying supernova}

The magnitudes of the host galaxy measured in the $R$ - and $I$ bands are approximately one magnitude fainter than what was determined from the GROND late-time photometric follow-up $\left(R_{\mathrm{FORS} 2}-r_{\mathrm{GROND}}^{\prime}=0.94 \pm 0.40 \mathrm{mag}\right.$ and $I_{\mathrm{FORS} 2}-i_{\mathrm{GROND}}^{\prime}=$ $0.94 \pm 0.35 \mathrm{mag}$ ) of the host (Fynbo et al. 2014). This could indicate a contribution from a bright underlying supernova (SN) 70 days ( $\approx 35$ days in rest-frame) after trigger during the GROND observations. If this is true, then the GROND magnitudes implies an observed $r^{\prime}$-band SN luminosty of approximately $1.5 \times$ $10^{39} \mathrm{erg} \mathrm{s}^{-1}$.

\subsection{Emission line measurements}

We were able to detect the prominent $\mathrm{H} \alpha$ and $\mathrm{H} \beta$ emission lines as well as emission from the $[\mathrm{OII}] \lambda \lambda 3726,3729$ doublet and the [O III] $\lambda 5007$ transition. To extract the line fluxes we fitted a Gaussian function to each line with the continuum set in small regions around the centroid of the fit $( \pm 30 \AA)$, free of telluricand sky-lines. Line widths were first fixed to a common value in the fits. The fit is dominated by the strongest line, $\mathrm{H} \alpha$. From the fit we then determined the FWHM for each line. This is converted to a velocity dispersion, $\sigma$, by subtracting the instrumental broadening, $\mathcal{R}\left(\mathrm{km} \mathrm{s}^{-1}\right)$, quadratically from the fitted FWHM as

$\sigma=\sqrt{F W H M^{2}-\mathcal{R}^{2}} /(2 \sqrt{2 \ln 2})$. 
K. E. Heintz et al.: Reconciling the steep extinction towards GRB 140506A

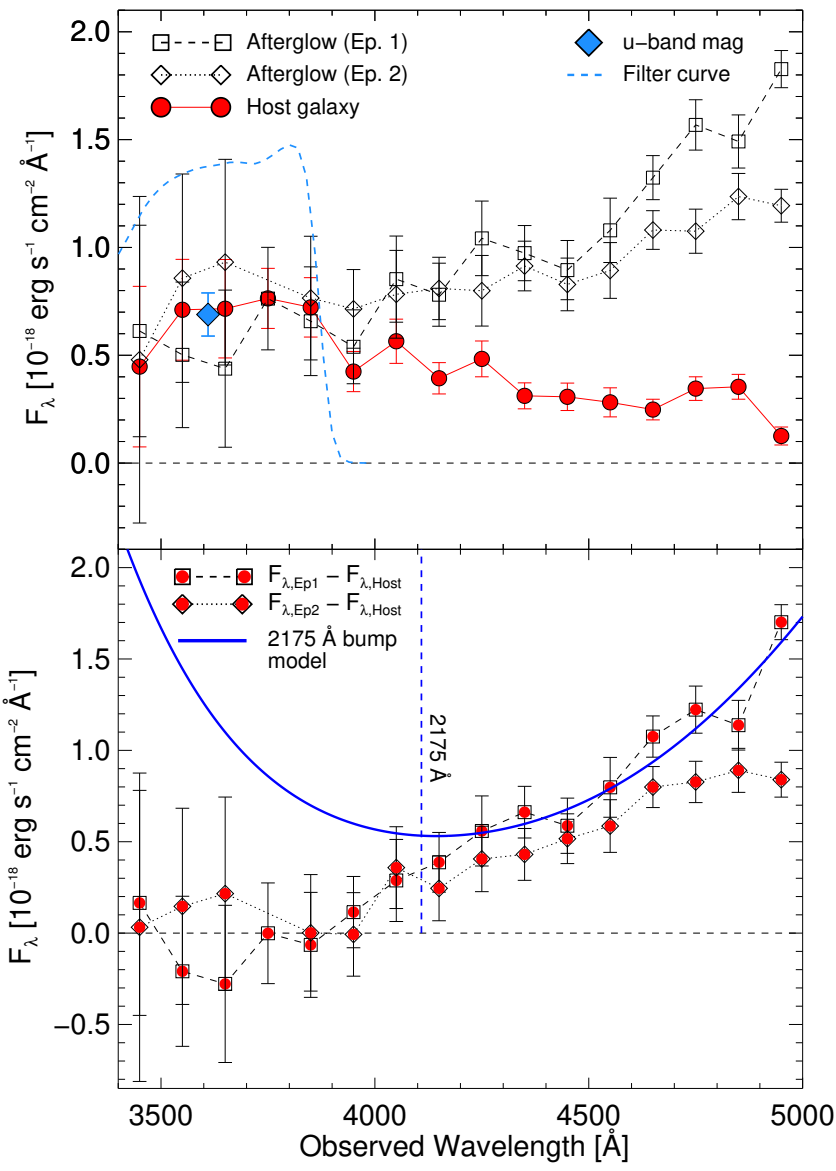

Fig. 3. Top panel: comparison of the spectra of the first and second epoch afterglow and the spectrum of the host galaxy at $\lambda<5000 \AA$, each binned by $100 \AA$, together with the measured VLT/FORS $2 u$-band magnitude of the host. The consistent lower flux in the first epoch afterglow spectrum below $4000 \AA$ is due to the host galaxy being partly outside of the slit (see Fig. 2). Bottom panel: first and second epoch afterglow spectra with the flux of the host galaxy subtracted. Overplotted is the $2715 \AA$ extinction bump model from Fynbo et al. (2014), which can now be securely excluded as the scenario causing the steep extinction.

Based on the strong $\mathrm{H} \alpha$ line we measure an emission-line redshift of $z=0.88905$ for the host galaxy, consistent with what has been reported in Fynbo et al. (2014) and Krühler et al. (2015). The results of the measured line fluxes and line widths are listed in Table 3 and shown in Fig. 4 with the best-fit Gaussian function.

\subsection{Attenuation of the host}

The observed $\mathrm{H} \alpha$ and $\mathrm{H} \beta$ emission line fluxes (the ratio of which is known as the Balmer decrement) provides us with the dust attenuation of the whole system toward the H II regions. The intrinsic, dust-corrected ratio of these two lines, $r_{\text {int }}$, assuming a case $\mathrm{B}$ recombination and an electron temperature of $10^{4} \mathrm{~K}$ and a density of $10^{2}-10^{4} \mathrm{~cm}^{-3}$, is $\mathrm{H} \alpha / \mathrm{H} \beta=2.87$ (Osterbrock 1989). Using the MW-type extinction curve of Pei (1992) with $R_{\mathrm{V}}=3.08$, we find that the attenuation at the wavelength of $\mathrm{H} \alpha$ and $\mathrm{H} \beta$ is $A_{\mathrm{H} \alpha}=0.82 \mathrm{mag}$ and $A_{\mathrm{H} \beta}=1.17 \mathrm{mag}$, for this specific extinction curve normalised to $A_{\mathrm{V}}=1 \mathrm{mag}$, respectively. We thus calculate the visual attenuation toward the H II regions
Table 3. Extracted emission-line fluxes.

\begin{tabular}{lccc}
\hline \hline Transition & Line flux $^{a}$ & $\sigma^{b}$ & $z$ \\
\hline$[\mathrm{O}$ II $] \lambda \lambda 3726,3729$ & $19.11 \pm 0.60$ & $50.8 \pm 4.7$ & $0.88905^{c}$ \\
$\mathrm{H} \beta$ & $3.27 \pm 0.73$ & $48.6 \pm 15.7$ & 0.88905 \\
{$[\mathrm{O}$ III $] \lambda 5007$} & $7.42 \pm 0.72$ & $48.0 \pm 13.0$ & 0.88905 \\
$\mathrm{H} \alpha$ & $16.47 \pm 0.60$ & $49.8 \pm 4.7$ & 0.88905 \\
\hline
\end{tabular}

Notes. The values are derived from the best-fit Gaussian functions, measured in a $\pm 30 \AA$ region around the centroid of each line. The listed errors include the propagating errors from the fit. ${ }^{(a)}$ Line fluxes are reported in units of $10^{-18} \mathrm{erg} \mathrm{cm}^{-2} \mathrm{~s}^{-1}$. ${ }^{(b)}$ Velocity dispersions are in units of $\mathrm{km} \mathrm{s}^{-1}$ and are corrected for the measured resolution in the respective arms. ${ }^{(c)}$ We only report one redshift and velocity dispersion, since the fit to the [O II] doublet was made with a fixed relation between the two components.

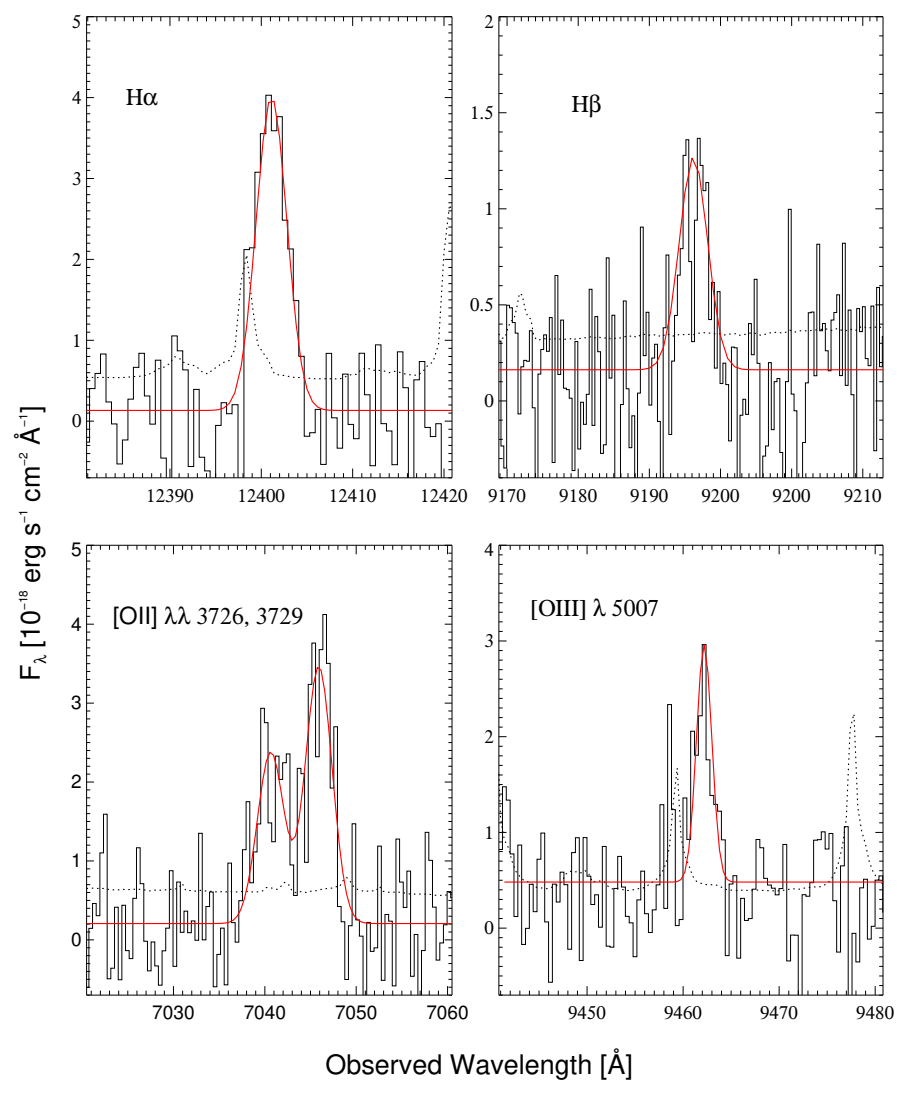

Fig. 4. Emission lines from the optimally-extracted 1D spectrum. In each panel the observed spectrum is shown by the solid line, the error spectrum by the dotted line and the best-fit Gaussian function by the solid, red line. The plotting region shows a zoom-in on the continuum region $( \pm 20 \AA)$.

from the Balmer decrement as

$A_{\mathrm{V}}=\frac{-2.5 \log \left(r_{\mathrm{obs}} / r_{\mathrm{int}}\right)}{A_{\mathrm{H} \alpha}-A_{\mathrm{H} \beta}}=1.74 \pm 0.41 \mathrm{mag}$,

where in our case, $r_{\mathrm{obs}}$ is found to be $(\mathrm{H} \alpha / \mathrm{H} \beta)_{\mathrm{obs}}=5.04 \pm 0.67$. We note that modelling the spectrum with an SMC-like extinction curve instead (also typically seen in GRB host galaxies; Schady et al. 2010) will not change the results significantly since there is little difference at the wavelength range of the Balmer lines, and that most extinction curves behave similar redwards of the $2175 \AA$ extinction bump. The MW extinction curve of 
Pei (1992) was also chosen to be consistent with Krühler et al. (2015). See Sect. 4 for further discussion on the reddening of the host and the comparison to other GRB host galaxies.

\subsection{Star formation rate}

To measure the star formation rate (SFR) of the host galaxy we calculated the intrinsic, dust-corrected luminosity of the $\mathrm{H} \alpha$ emission line using the luminosity distance computed from the redshift, $z=0.88905$. This yields a de-reddened line luminosity of $L(\mathrm{H} \alpha)=(2.61 \pm 0.09) \times 10^{41} \mathrm{erg} \mathrm{s}^{-1}$, where the applied dust-correction was calculated as

$L_{\text {dered }}=L_{\text {int }} \times 10^{\left(0.4 \times A_{\lambda}\right)}$.

Using the relation from Kennicutt (1998) and assuming a Salpeter initial mass function (IMF) we calculate a dustcorrected SFR of

$S F R(\mathrm{H} \alpha)=7.9 \times 10^{-42} L(\mathrm{H} \alpha)_{\mathrm{dered}}=2.14 \pm 0.07 M_{\odot} \mathrm{yr}^{-1}$,

where, assuming a Chabrier IMF instead, this can be converted to $S F R(\mathrm{H} \alpha)_{\text {Chab }}=1.34 \pm 0.04 M_{\odot} \mathrm{yr}^{-1}$ (Treyer et al. 2007). For consistency with Krühler et al. (2015) we refer only to the latter value in Sect. 4 in our comparison.

\subsection{Metallicity}

To infer the emission-line metallicity of the host galaxy it is common practice to use the strong-line ratios; [N II $] /[\mathrm{O}$ II $](\mathrm{N} 2 \mathrm{O} 2)$, $([\mathrm{O}$ II $] \lambda 3727+[\mathrm{O}$ III $] \lambda 4959+[\mathrm{O}$ III $] \lambda 5007) / \mathrm{H} \beta\left(R_{23}\right),[\mathrm{N} \mathrm{II}] / \mathrm{H} \alpha$ $(\mathrm{N} 2)$ and $([\mathrm{O} \mathrm{II} / \mathrm{H} \beta) /([\mathrm{N} \mathrm{II}] / \mathrm{H} \alpha)(\mathrm{O} 3 \mathrm{~N} 2)$. A detailed discussion on how the strong-line diagnostics can be used as metallicity indicators and a comparison of these is given in Kewley \& Ellison (2008). In the following we assume a solar metallicity of $12+$ $\log (\mathrm{O} / \mathrm{H})=8.69$ (Asplund et al. 2009).

Due to the non-detection of [N II] in our case (Fig. 5), we are only able to determine upper limits from the aforementioned strong-line ratios from the $3 \sigma$ upper limit measured from the continuum at the wavelength of [N II]. From the dust-corrected emission-line fluxes (Eq. (3)) we compute upper limits on the oxygen abundances of $12+\log (\mathrm{O} / \mathrm{H})<8.46$ for N2O2 (Kewley \& Dopita 2002), $12+\log (\mathrm{O} / \mathrm{H})<8.37$ for N2 (Pettini \& Pagel 2004) and $12+\log (\mathrm{O} / \mathrm{H})<8.67$ for O3N2 (Pettini \& Pagel 2004) corresponding to upper limits of $0.59,0.49 \& 0.95 Z / Z_{\odot}$, respectively. The errors and upper limits on all derivations include the scatter in the relations listed in Kewley \& Ellison (2008).

To alleviate the limitations from the non-detection of [N II] we make use of the fact that it is also possible to estimate the metallicity based solely on the strong oxygen nebular lines (Pilyugin 2001; Pilyugin \& Thuan 2005), the so-called $P$ method. The authors advocate that the physical properties of the H II region can be estimated via the excitation parameter, $P$, here defined as $P=R_{3} /\left(R_{2}+R_{3}\right)$, where $R_{2}=[\mathrm{O} \mathrm{II}] / \mathrm{H} \beta$ and $R_{3}=([\mathrm{O} \mathrm{III}] \lambda 4959+[\mathrm{O} \mathrm{III}] \lambda 5007) / \mathrm{H} \beta$. Since we do not detect $[\mathrm{O}$ III $] \lambda 4959$ we use that $f_{[\mathrm{OIII}]} \lambda 4959=1 / 3 \times f_{[\mathrm{OIII}]} \lambda 5007$ (Storey \& Zeippen 2000).

Following the above definitions, we derive an excitation parameter of $P=0.21 \pm 0.03$. To compute the metallicity we use the relation

$12+\log (\mathrm{O} / \mathrm{H})=\frac{R_{3}+106.4 P+106.8 P^{2}-3.40 P^{3}}{17.72 P+6.60 P^{2}+6.95 P^{3}-0.302 R_{3}}$,

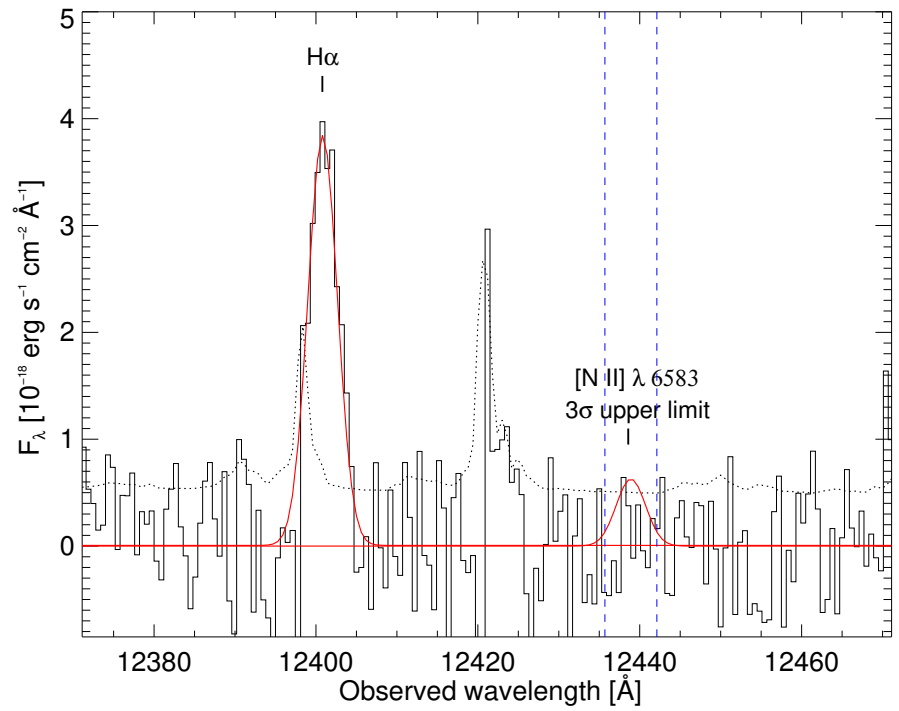

Fig. 5. The $3 \sigma$ flux limit of [N II] measured from the continuum compared to $\mathrm{H} \alpha$. We assumed a Gaussian shape of the line profile and measured the limit at the FWHM (dashed blue lines) of that of $\mathrm{H} \alpha$. The total flux of the continuum at [N II] was then computed from the flux encompassed in this region.

from Pilyugin \& Thuan (2005). Using the value for the excitation parameter found above and the ratio $R_{3}=2.81 \pm 0.45$ this yields an oxygen abundance of $12+\log (\mathrm{O} / \mathrm{H})=8.23 \pm 0.16$, corresponding to $35 \%$ solar metallicity $\left(Z=0.35_{-0.11}^{+0.15} Z_{\odot}\right)$. The expected scatter of 0.1 dex in the relation is included in the calculation. This is consistent with the previous upper limits. We will only refer to this value in the remainder of this paper.

\section{Discussion}

\subsection{Broad-band SED of the dusty host}

To constrain the physical origin of the steep extinction curve seen towards the sightline of GRB 140506A, we first model the SED of the host galaxy. Fitting the observed five broad-band magnitudes reported in Table 1 in LePhare ${ }^{3}$ (Arnouts et al. 1999; Ilbert et al. 2006) yields the host galaxy parameters listed in Table 4 , with a $\chi^{2}=0.98$, and the best-fit stellar population synthesis model shown in Fig. 6. We obtained the best fit SED by fixing the redshift to $z=0.88905$ and used a grid of stellar evolution models with varying star formation time scales, age of stellar population and extinction assuming the models from Bruzual \& Charlot (2003) based on an IMF from Chabrier (2003) and a Calzetti extinction curve (Calzetti et al. 2000).

The best-fit visual attenuation, $A_{\mathrm{V}}^{\mathrm{SED}}$, and star-formation rate from the broad-band SED agree well with that determined from the emission-line diagnostics. The stellar mass computed by the fit is mainly dominated by the $3.6 \mu \mathrm{m}$ Spitzer photometric data point.

It is evident that the host galaxy does not follow an extinction curve as steep as that seen towards the GRB explosion site. Running the SED fit with the new extinction curve (see next section) in steps of $E(B-V)=[0.0,0.05, \ldots, 1.0]$ we find a best-fit with no attenuation, that is all models with the steep extinction curve implemented provides worse fit to the data. As a conclusion, the steep extinction curve toward the afterglow must be sightline

\footnotetext{
3 http://www. cfht.hawaii.edu/ arnouts/LEPHARE/lephare. html
} 


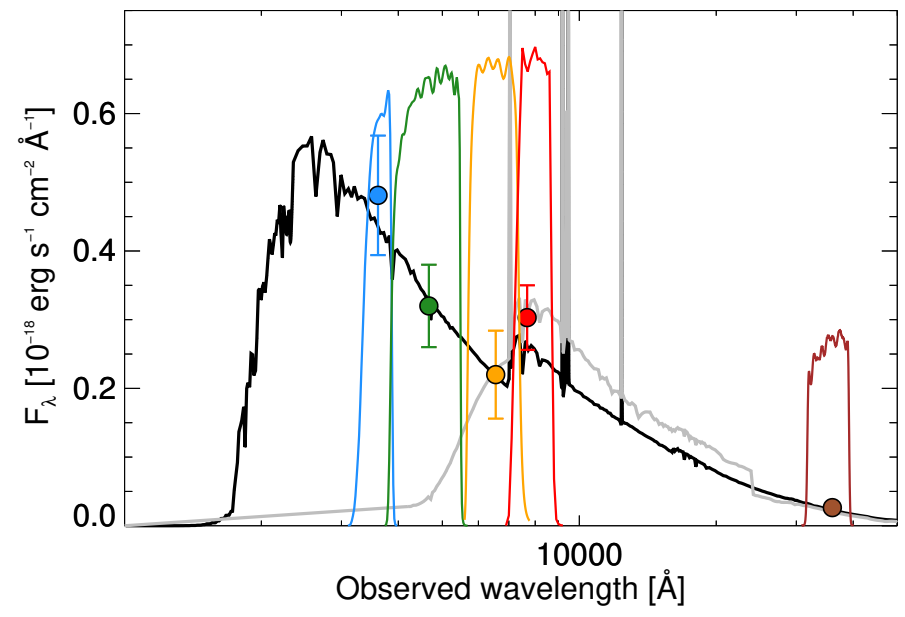

Fig. 6. SED of the host galaxy of GRB 140506A. Overplotted is the best-fit stellar population synthesis model of a galaxy with a $20 \mathrm{Myr}$ old stellar population at a fixed redshift of $z=0.88905$, reddened by $A_{\mathrm{V}}=1.62 \mathrm{mag}$ (black line) following the extinction curve of Calzetti et al. (2000). Overplotted is the same intrinsic SED but reddened by the steep extinction curve seen towards the afterglow instead (grey line) but without the rise in flux at wavelengths below $4000 \AA$ (see text). Also shown are the filter transmission curves for the corresponding five photometric points. The data do not suggest an atypical steep extinction curve for the host, similar to the afterglow sightline.

Table 4. Host galaxy parameters from SED fitting.

\begin{tabular}{lr}
\hline \hline Extinction law & Calzetti et al. (2000) \\
Reddening $A_{\mathrm{V}}[\mathrm{mag}]$ & $1.62 \pm 0.21$ \\
$S F R\left[M_{\odot} \mathrm{yr}^{-1}\right]$ & $1.18 \pm 0.28$ \\
Age $[\mathrm{Gyr}]$ & $0.02_{-0.02}^{+0.07}$ \\
$\log \left(M_{*} / M_{\odot}\right)$ & $8.35 \pm 0.26$ \\
\hline
\end{tabular}

dependent and this is evidence for the occurrance of only locally steep extinction curves. The dust properties of the intermediate environment in which the GRB is embedded, strongly imprinted on the afterglow SED, are therefore not representative of the dust properties of the host galaxy as a whole.

\subsection{Modelling the extinction curve}

To further confine the shape of the extinction curve we subtract the flux of the host galaxy from the best-fit SED from the first epoch afterglow. To estimate the reddening we assume that the intrinsic spectrum follows a power-law, $F_{\lambda} \propto \lambda^{-\beta}$, with $\beta=1.75$ as derived from the XRT spectrum with a break between the $\mathrm{X}$-rays and optical bands of $\Delta \beta=0.5$ (following the same procedure as Fynbo et al. 2014). We then run a non-linear least squares curve fitting on the host-subtracted afterglow spectrum using MPFIT in IDL with the parametrization of Fitzpatrick \& Massa (2007), which models the extinction curve through a set of nine parameters. It basically contains two components, one describing the UV linear part of the extinction specified by the parameters $c_{1}$ (intercept) and $c_{2}$ (slope) and the parameters $c_{4}$ and $c_{5}$ provide the far-UV curvature and the other is the Drude component describing the $2175 \AA$ bump by the parameters $c_{3}$ (bump strength), $x_{0}$ (central wave number) and $\gamma$ (width of the bump). The last two parameters are the visual extinction, $A_{\mathrm{V}}$, and the total-to-selective reddening, or the steepness of the reddening law, $R_{\mathrm{V}}$.
Setting all nine parameters free we find a best-fit almost identical to the extreme $2175 \AA$ extinction bump proposed by Fynbo et al. (2014). However, having excluded any signs of a bump in the extinction curve we instead run the fit assuming $c_{3}=0$, effectively removing the Drude profile representing the bump in the model. We then find the best-fit values of: $c_{1}=-0.93, c_{2}=3.13, c_{4}=3.30, c_{5}=2.15, R_{\mathrm{V}}=5.12$ and $A_{\mathrm{V}}=1.04 \mathrm{mag}$, resulting in the extinction curve shown in Fig. 7 and defined as

$$
\frac{A_{\lambda}}{A_{V}}= \begin{cases}\frac{1}{5.12}(-0.93+3.13 x)+1 & x<c_{5} \\ \frac{1}{5.12}\left(-0.93+3.13 x+3.30(x-2.15)^{2}\right)+1 & x>c_{5},\end{cases}
$$

where $x=\lambda^{-1}$ in units of $\mu \mathrm{m}^{-1}$. Most notable are the flat or grey total-to-selective extintion parameter, $R_{\mathrm{V}}$, and the parameter describing the far-UV curvature component, $c_{4}$. Comparing these to a simple SMC-type extinction curve as modelled by Gordon et al. (2003) reveals that a typical steeper reddening law, commonly defined by a small value of $R_{\mathrm{V}}$, is inadequate in reproducing the strong drop. Instead a flat extinction curve is required, mainly to reproduce the relatively blue part of the spectrum at wavelengths above $8000 \AA$, with the roughly seven times larger value of $c_{4}$ compared to that of the SMC (Gordon et al. 2003) modelling the steep drop. The parameters describing the linear component of the UV extinction, $c_{1}$ and $c_{2}$, also differentiate significantly compared to the relation $c_{1}=2.09-2.84 c_{2}$ found by Fitzpatrick \& Massa (2007), where we would expext $c_{1}=-6.80$ (best-fit $c_{1}=-0.93$ ) based on the $c_{2}$ parameter.

From the above derived parametrization of the extinction we attempt to recover the relative reddening curve as well. By again assuming the XRT spectrum with a cooling break as the instrinsic afterglow spectrum, we normalise this power-law to the $K$-band (at roughly $20000 \AA$ in regions with no telluric absorption) and then we calculate the relative reddening, $k(\lambda)$, as

$k(\lambda)=-2.5 \log \left(D_{\lambda} / I_{\lambda}\right)$,

where $D_{\lambda}$ denotes the observed data and $I_{\lambda}$ refers to the intrinsic spectrum. The resulting reddening curve is shown in Fig. 8 where we also compare it to the standard extinction curves of the Local Group (that of the MW, the SMC and the LMC). It is evident that the standard reddening laws do not provide a good fit to the data, except in the reddest part of the spectrum.

\subsection{Comparison to GRB hosts at $z<1$}

Another potential clue to understand why the afterglow of GRB 140506A is so different from the typical SED of GRB afterglows, is to compare the properties of the host galaxy to other GRB hosts at similar redshifts. We use a subsample of 20 GRB host galaxies at $z<1$ with metallicity measurements extracted from the full sample of 96 GRB-selected galaxies presented in Krühler et al. (2015). We caution that this is not an unbiased sample but merely a compilation of GRB host galaxies observed with X-shooter. In Fig. 9 we show the physical properties of this subsample and compare these GRB host galaxies with GRB 140506A. It is evident that this particular host galaxy is a standard GRB host in terms of star formation rate, velocity dispersion and metallicity but with a large, though not abnormal, visual attenuation, $A_{\mathrm{V}}$. 


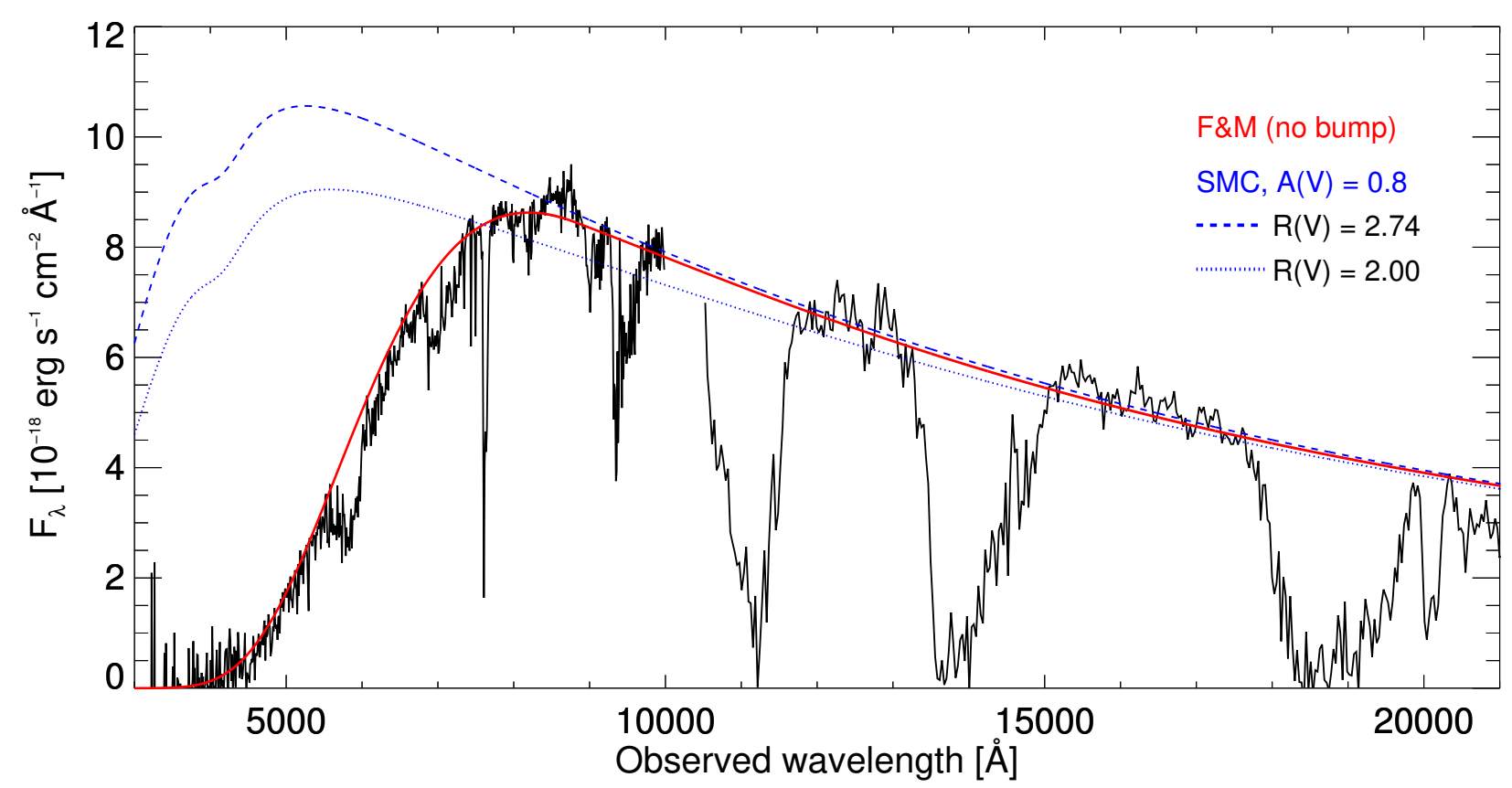

Fig. 7. First epoch afterglow spectra with the flux of the host galaxy from the best-fit SED subtracted. Overplotted is the best-fit Fitzpatrick \& Massa (2007) parametrization of the extinction curve with $c_{3}=0$ (no bump) shown by the red solid line. We assume an underlying power-law with slope set by a fit to the X-ray afterglow and assuming a $\Delta \beta=0.5$ cooling break between the X-rays and the optical. Regions affected by telluric absorption are removed from the fit. Also plotted is the extinction curve of the SMC as described by Gordon et al. (2003); blue dashed line) and with an decreased total-to-selective reddening, $R_{\mathrm{V}}$ (blue dotted line). These examples proves to show that the extinction can not simply be modelled by a standard or even steeper SMC-type reddening law. The parameters of the best-fit extinction curve diverges significantly from that known from the local analogs, most notable are the $c_{4}$ and $c_{5}$ values representing the far-UV curvature component, but also the flat value of $R_{\mathrm{V}}$. These are required to model the extreme drop without assuming a bump in the extinction curve in combination with the otherwise blue spectrum.

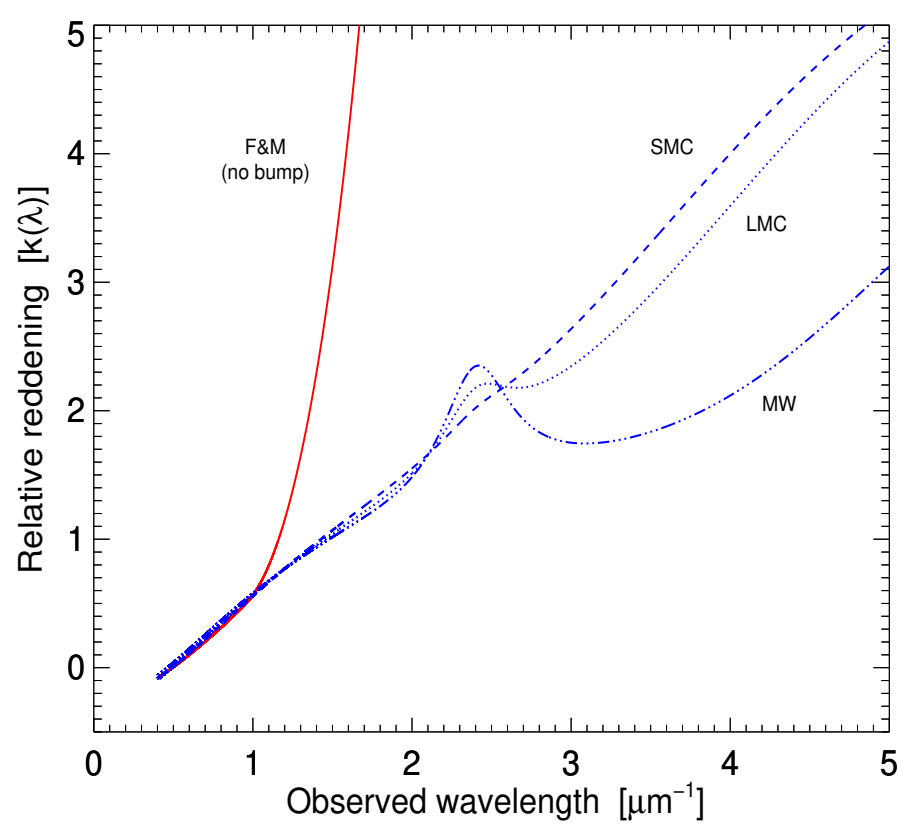

Fig. 8. Reddening curves for the first epoch afterglow spectra with the flux of the host galaxy from the best-fit SED subtracted. Since we do not know the instrinsic brightness of the afterglow the data has been normalised to the flux in the $K$-band $(\sim 20000 \AA)$. The reddening curves, described by $k(\lambda)$, thus only indicate the relative reddening and not the total extinction. It is clearly shown how the extinction curves of the Local Group, here the MW, SMC and LMC, can only match the best-fit reddening law from the Fitzpatrick \& Massa (2007) parametrization at wavelengths longer than $8000 \AA$ (observers frame), at which point the steep extinction curve diverges significantly.

\subsection{Comparison to $S N e$ and AGNs}

One of the main differences between observed GRB and SN samples is that the explosion sites of GRBs are found much closer to the brighter regions of their respective host galaxies (Fruchter et al. 2006; Lyman et al. 2017).

Numerous more cases of $\mathrm{SNe}$ with steep extinction curves are observed (e.g. Krisciunas et al. 2006; Nobili \& Goobar 2008; Folatelli et al. 2010), which are argued could imply a grain size that is small in the specific line of sights compared with the average value for the local ISM (Elias-Rosa et al. 2006, 2008) or a dominating multiple scattering process (Goobar 2008). See also Amanullah et al. (2014) for a prime example of a steep extinction curve seen towards SN 2014J in M82. Again we wish to highlight that in this specific case the multiple scattering scenario was ruled out (Johansson et al. 2017). Hutton et al. (2014) were able to constrain the global reddening law of M82, showing that standard MW-type extinction is preferred for the galaxy as a whole, which is further evidence that steep extinction is only seen locally. In these studies it is argued that the smaller grain size causing the steep extinction could be fragmentations of larger dust grains disolved due to the large radiation field.

A similar steep extinction curve is observed toward several dust-reddened quasars as well. Fynbo et al. (2013) found that $\sim 30 \%$ of the quasars in their sample were reddened following an extinction curve much steeper than what is seen in the local analogs; MW, SMC or LMC (see also Leighly et al. 2014, for a specific example towards the AGN Mrk 231). A similar type of extinction is also seen (but to a lesser extent) in the High $A_{\mathrm{V}}$ Quasar (HAQ; Krogager et al. 2015) and the extended HAQ (eHAQ; Krogager et al. 2016) surveys. A subsample of 16 intrinsically reddened quasars from the HAQ survey were examined 


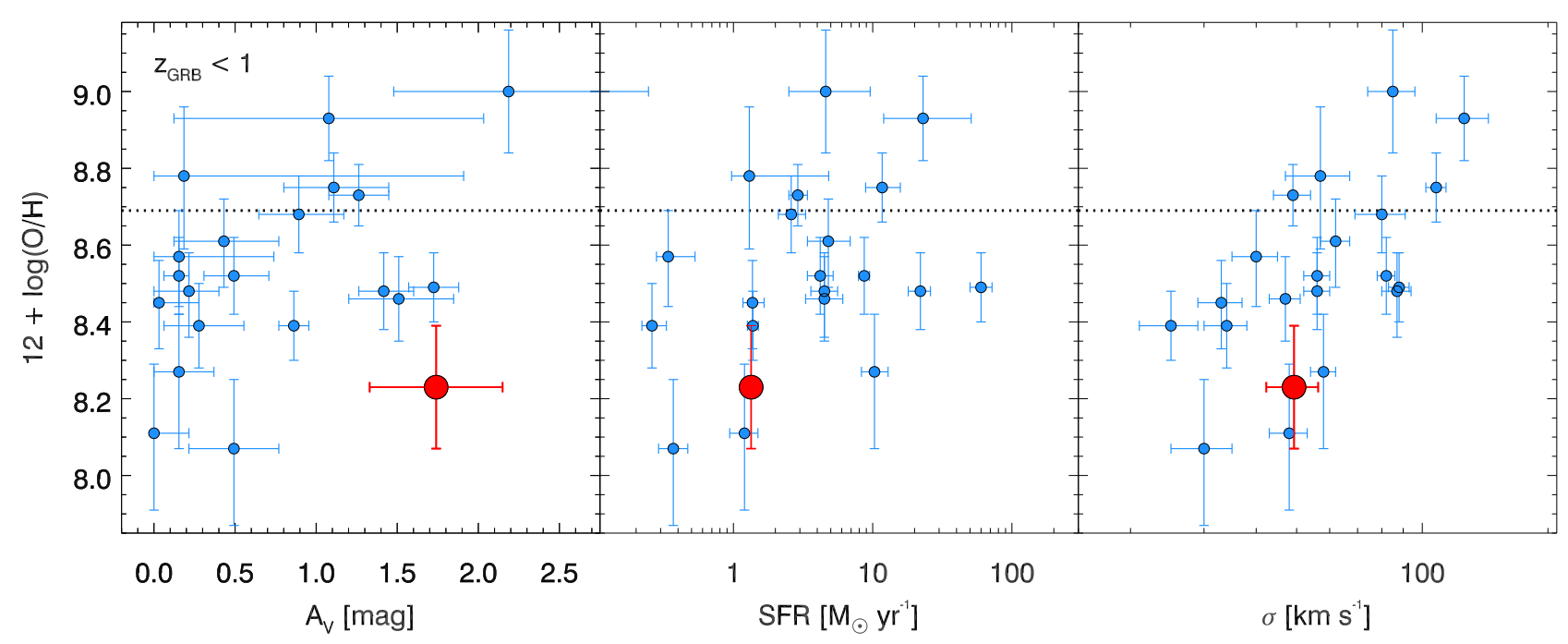

Fig. 9. Comparison of the host galaxy properties of GRB 140506A (large red dot) to 20 other GRB host galaxies at $z<1$ (small blue dots) with metallicity measurements from Krühler et al. (2015). From left to right the metallicity of each individual host galaxy is shown as a function of: $A_{\mathrm{V}}$, SFR and $\sigma$, measured from emission line diagnostics. The dotted, horizontal line denotes the solar metallicity abundunce (Asplund et al. 2009). The physical properties of the host galaxy of GRB 140506A is inconspicuous, compared to the other GRB host galaxies at $z<1$.

by Zafar et al. (2015), who found a weighted mean value of $R_{\mathrm{V}}=2.4$, much steeper than that of the SMC which has a totalto-selective extinction ratio around $R_{\mathrm{V}} \sim 2.9$. We note that even these steep extinction curves do not provide a good fit to the data, due to the still close resemblance to that of the SMC.

\subsection{Variability of the flux drop}

The above cases further suggests that the steep extinction curve observed towards GRB 140506A is not a rare phenomenon, but is more likely attributed to the level of fragmentation of the dust grains surrounding the explosion site. More curios is the temporal variability of the first to the second epoch afterglow spectrum. From a study of the dynamics of large dust grains in molecular clouds, Hopkins \& Lee (2016) show that grains with sizes $a>0.01 \mu \mathrm{m}$ can exhibit large fluctuations in the local dustto-gas ratio. They conclude that small dust grains can be much more clumped than larger dust grains, critical for dust growth and shattering.

If that is the case for the circumburst medium then this might explain the temporally varying afterglow SED. Due to the decrease of the relativistic beaming as the blast wave slows down, the emitting area of the afterglow expands as $(\gamma c t)^{2}$ (see also the discussion in Fynbo et al. 2014), which yields a factor of six increase in the glowing region producing the afterglow between 8.8 and $33 \mathrm{~h}$ post-burst. If the probed distribution of small and large dust grains is different for such different beam sizes, then this will have an effect on the observed extinction towards the line of sight to the GRB at the two different epochs.

\section{Conclusions}

We have analysed the host galaxy of the mysterious GRB 140506A afterglow, showing an extreme flux-drop below $8000 \AA$ observers frame (4000 ̊ rest-frame) in the sightline towards the GRB explosion site (Fynbo et al. 2014). This is atypical to the blue power-law SEDs commonly observed for GRB afterglows (e.g. Fynbo et al. 2009). We found that the explosion site of the GRB occurred at a projected distance of $\approx 4 \mathrm{kpc}$ from the galactic centre, in a faint region in the outskirts of the host galaxy, while most GRBs are observed much closer to the centre or in the brightest regions of their respective host galaxies (Fruchter et al. 2006; Lyman et al. 2017).

We found the host galaxy to be moderately forming stars at a rate of $1.34 \pm 0.04 M_{\odot} \mathrm{yr}^{-1}$. Employing the $P$ method we derived a metallicity of $12+\log (\mathrm{O} / \mathrm{H})=8.23 \pm 0.16\left(Z=0.35_{-0.11}^{+0.15} Z_{\odot}\right)$. This is in good agreement with the $Z-$ SFR and $Z-\sigma$ relations found for GRB host galaxies at $z<1$ (Krühler et al. 2015). The host galaxy can be characterised by a large visual attenuation of $A_{\mathrm{V}}=1.74 \pm 0.41 \mathrm{mag}$ based on emission line diagnostics.

We excluded the scenario where the rise in flux observed at $<4000 \AA$ in the afterglow spectrum was due to an extreme $2175 \AA$ extinction bump, a feature known from the local LMC and the MW, and showed that it was merely a representation of the dominating host galaxy light at this wavelength. There does not seem to be any similarity between the extinction law of the afterglow and the best-fit for the host galaxy which suggests that the steep extinction seen in the afterglow SED is sightline dependent and only a local effect. The $A_{\mathrm{V}}$ 's measured in both cases are high, with a best-fit afterglow extinction of $A_{\mathrm{V}}=1.04$ mag. This effect could be explained by the intense radiation field from the GRB only fragmenting (in contrast to fully destroying) the surrounding dust particles causing a local only steep extinction.

We modelled the relative reddening and showed that all the standard extinction curves as seen in the Local Group (MW, SMC and LMC) are inadequate in descring the steep drop in combination with the relatively blue underlying power-law spectrum. A similar non-standard, steep extinction curve is observed toward a few other rare cases of GRBs but also in several other types of enigmatic astrophysical objects, such as AGNs and SNe. The phenomenon of steep extinction curves does not seem to be rare globally and also hint that this could contribute (at least partly) to the large fraction of dark GRBs, defined by no optically detected afterglows. A new picture of the dust properties in high energy environment seems to be emerging. The extinction curves with non-local analogs appear to be an important clue to resolve this.

Acknowledgements. We would like to thank the anonymous referee whose comments greatly improved the quality of the paper and arrived in a timely manner. We would also like to thank C. Gall, D. Malesani and B. T. Draine for insightful 
discussions on the conclusions of the paper. K.E.H. and P.J. acknowledge support by a Project Grant (162948-051) from The Icelandic Research Fund. The research leading to these results has received funding from the European Research Council under the European Union's Seventh Framework Program (FP7/20072013)/ERC Grant agreement No. EGGS-278202. T.K. acknowledges support through the Sofja Kovalevskaja Award to P. Schady from the Alexander von Humboldt Foundation of Germany. L.C. is supported by YDUN grant DFF 4090-00079. D.X. acknowledges the support by the One-Hundred-Talent Program of the Chinese Academy of Sciences (CAS), by the Strategic Priority Research Program "Multi-wavelength Gravitational Wave Universe" of the CAS (No. XDB23000000), and by the National Natural Science Foundation of China under grant 11533003 .

\section{References}

Amanullah, R., Goobar, A., Johansson, J., et al. 2014, ApJ, 788, L21 Appenzeller, I., Fricke, K., Fürtig, W., et al. 1998, The Messenger, 94, 1 Arnouts, S., Cristiani, S., Moscardini, L., et al. 1999, MNRAS, 310, 540 Asplund, M., Grevesse, N., Sauval, A. J., \& Scott, P. 2009, ARA\&A, 47, 481

Bloom, J. S., Kulkarni, S. R., \& Djorgovski, S. G. 2002, AJ, 123, 1111 Bruzual, G., \& Charlot, S. 2003, MNRAS, 344, 1000

Buchner, J., Schulze, S., \& Bauer, F. E. 2017, MNRAS, 464, 4545

Calzetti, D., Armus, L., Bohlin, R. C., et al. 2000, ApJ, 533, 682

Campana, S., Thöne, C. C., de Ugarte Postigo, A., et al. 2010, MNRAS, 402, 2429

Chabrier, G. 2003, PASP, 115, 763

Covino, S., Melandri, A., Salvaterra, R., et al. 2013, MNRAS, 432, 1231

De Cia, A., Ledoux, C., Savaglio, S., Schady, P., \& Vreeswijk, P. M. 2013, A\&A, 560, A88

Elíasdóttir, Á., Fynbo, J. P. U., Hjorth, J., et al. 2009, ApJ, 697, 1725

Elias-Rosa, N., Benetti, S., Cappellaro, E., et al. 2006, MNRAS, 369, 1880

Elias-Rosa, N., Benetti, S., Turatto, M., et al. 2008, MNRAS, 384, 107

Fitzpatrick, E. L., \& Massa, D. 2007, ApJ, 663, 320

Folatelli, G., Phillips, M. M., Burns, C. R., et al. 2010, AJ, 139, 120

Friis, M., De Cia, A., Krühler, T., et al. 2015, MNRAS, 451, 167

Fruchter, A. S., Levan, A. J., Strolger, L., et al. 2006, Nature, 441, 463

Fynbo, J. P. U., Starling, R. L. C., Ledoux, C., et al. 2006, A\&A, 451, L47

Fynbo, J. P. U., Jakobsson, P., Prochaska, J. X., et al. 2009, ApJS, 185, 526

Fynbo, J. P. U., Krogager, J.-K., Venemans, B., et al. 2013, ApJS, 204, 6

Fynbo, J. P. U., Krühler, T., Leighly, K., et al. 2014, A\&A, 572, A12

Gehrels, N., Ramirez-Ruiz, E., \& Fox, D. B. 2009, ARA\&A, 47, 567

Goldoni, P., Royer, F., François, P., et al. 2006, in Society of Photo-Optical Instrumentation Engineers (SPIE) Conference Series, Proc. SPIE, 6269, $62692 \mathrm{~K}$

Gompertz, B. P., Burrows, D. N., Cenko, S. B., et al. 2014, GRB Coordinates Network, 16214

Goobar, A. 2008, ApJ, 686, L103

Gordon, K. D., Clayton, G. C., Misselt, K. A., Landolt, A. U., \& Wolff, M. J. 2003, ApJ, 594, 279

Greiner, J., Bornemann, W., Clemens, C., et al. 2008, PASP, 120, 405

Hartoog, O. E., Malesani, D., Fynbo, J. P. U., et al. 2015, A\&A, 580, A139
Hopkins, P. F., \& Lee, H. 2016, MNRAS, 456, 4174

Hutton, S., Ferreras, I., Wu, K., et al. 2014, MNRAS, 440, 150

Ilbert, O., Arnouts, S., McCracken, H. J., et al. 2006, A\&A, 457, 841

Jakobsson, P., Hjorth, J., Fynbo, J. P. U., et al. 2004, A\&A, 427, 785

Japelj, J., Covino, S., Gomboc, A., et al. 2015, A\&A, 579, A74

Johansson, J., Goobar, A., Kasliwal, M. M., et al. 2017, MNRAS, 466, 3442

Kennicutt, Jr., R. C. 1998, ARA\&A, 36, 189

Kewley, L. J., \& Dopita, M. A. 2002, ApJS, 142, 35

Kewley, L. J., \& Ellison, S. L. 2008, ApJ, 681, 1183

Krisciunas, K., Prieto, J. L., Garnavich, P. M., et al. 2006, AJ, 131, 1639

Krogager, J.-K., Geier, S., Fynbo, J. P. U., et al. 2015, ApJS, 217, 5

Krogager, J.-K., Fynbo, J. P. U., Heintz, K. E., et al. 2016, ApJ, 832, 49

Krühler, T., Küpcü Yoldaş, A., Greiner, J., et al. 2008, ApJ, 685, 376

Krühler, T., Fynbo, J. P. U., Geier, S., et al. 2012, A\&A, 546, A8

Krühler, T., Malesani, D., Fynbo, J. P. U., et al. 2015, A\&A, 581, A125

Ledoux, C., Vreeswijk, P. M., Smette, A., et al. 2009, A\&A, 506, 661

Leighly, K. M., Terndrup, D. M., Baron, E., et al. 2014, ApJ, 788, 123

Li, L.-X. 2008, MNRAS, 388, 1487

Lyman, J. D., Levan, A. J., Tanvir, N. R., et al. 2017, MNRAS, 467, 1795

Modigliani, A., Goldoni, P., Royer, F., et al. 2010, in Observatory Operations: Strategies, Processes, and Systems III, Proc. SPIE, 7737, 773728

Nobili, S., \& Goobar, A. 2008, A\&A, 487, 19

Oke, J. B. 1974, ApJS, 27, 21

Osterbrock, D. E. 1989, Astrophysics of gaseous nebulae and active galactic nuclei (University Science Books)

Pei, Y. C. 1992, ApJ, 395, 130

Perley, D. A., Bloom, J. S., Butler, N. R., et al. 2008, ApJ, 672, 449

Perley, D. A., Morgan, A. N., Updike, A., et al. 2011, AJ, 141, 36

Perley, D. A., Krühler, T., Schulze, S., et al. 2016, ApJ, 817, 7

Pettini, M., \& Pagel, B. E. J. 2004, MNRAS, 348, L59

Pilyugin, L. S. 2001, A\&A, 369, 594

Pilyugin, L. S., \& Thuan, T. X. 2005, ApJ, 631, 231

Planck Collaboration, Ade, P. A. R., Aghanim, N., et al. 2014, A\&A, 571, A16

Prochaska, J. X., Chen, H.-W., Dessauges-Zavadsky, M., \& Bloom, J. S. 2007, ApJ, 666, 267

Prochaska, J. X., Sheffer, Y., Perley, D. A., et al. 2009, ApJ, 691, L27

Savaglio, S., \& Fall, S. M. 2004, ApJ, 614, 293

Schady, P., Page, M. J., Oates, S. R., et al. 2010, MNRAS, 401, 2773

Schady, P., Dwelly, T., Page, M. J., et al. 2012, A\&A, 537, A15

Schlafly, E. F., \& Finkbeiner, D. P. 2011, ApJ, 737, 103

Sparre, M., Hartoog, O. E., Krühler, T., et al. 2014, ApJ, 785, 150

Starling, R. L. C., Willingale, R., Tanvir, N. R., et al. 2013, MNRAS, 431, 3159

Storey, P. J., \& Zeippen, C. J. 2000, MNRAS, 312, 813

Thöne, C. C., Fynbo, J. P. U., Goldoni, P., et al. 2013, MNRAS, 428, 3590

Tody, D. 1993, in Astronomical Data Analysis Software and Systems II, eds.

R. J. Hanisch, R. J. V. Brissenden, \& J. Barnes, ASP Conf. Ser., 52, 173

Treyer, M., Schiminovich, D., Johnson, B., et al. 2007, ApJS, 173, 256

Vernet, J., Dekker, H., D’Odorico, S., et al. 2011, A\&A, 536, A105

Watson, D. 2009, in Cosmic Dust - Near and Far, eds. T. Henning, E. Grün, \& J. Steinacker, ASP Conf. Ser., 414, 277

Zafar, T., Watson, D., Fynbo, J. P. U., et al. 2011, A\&A, 532, A143

Zafar, T., Watson, D., Elíasdóttir, Á., et al. 2012, ApJ, 753, 82

Zafar, T., Møller, P., Watson, D., et al. 2015, A\&A, 584, A100 